\title{
Modifying Epoxy Resins to Resist Both Fire and Water
}

\author{
Xiaoyang Wang, ${ }^{\dagger, \S}$ Qiaoran Zhang, ${ }^{\dagger, \S}$ Xia Zhang, ${ }^{*}{ }^{\dagger} \odot$ Zhiwei Li, ${ }^{\dagger} \odot$ Ivan P. Parkin, ${ }^{\ddagger}$ and Zhijun Zhang ${ }^{\dagger}$ \\ $\dagger$ Engineering Research Center for Nanomaterials, Henan University, Kaifeng 475004, China \\ ${ }^{\ddagger}$ Department of Chemistry, University College London, 20 Gordon Street, London WC1H 0AJ, U.K.
}

ABSTRACT: In this work, we converted inherently hydrophilic and flammable epoxy resins to resist both fire and water. This was achieved by integrating surface modified flame-retardant $\mathrm{CoFe}_{2} \mathrm{O}_{4}$ nanoparticles into the epoxy resin. The modified $\mathrm{CoFe}_{2} \mathrm{O}_{4}$ nanoparticles reduce the peak heat release rate, peak smoke production release and $\mathrm{CO}$ production in the $\mathrm{CoFe}_{2} \mathrm{O}_{4}$ /epoxy resin nanocomposite by 39.6, 41.6, and $61.3 \%$, respectively. The obtained flame retardant $\mathrm{CoFe}_{2} \mathrm{O}_{4} / \mathrm{EP}$ items demonstrate excellent durability with constant superhydrophobicity even after high external pressure, knife-scratch, and mechanical abrasion. In addition, the obtained items demonstrate outstanding robust water-repellent properties after facing longterm exposure to extremely corrosive liquids and also show self-cleaning properties in air and under oil.

\section{INTRODUCTION}

Superhydrophobic surfaces have gained more attention in the scientific research area and in our daily life, because of their unique properties such as oil/water separation, ${ }^{1-3}$ selfcleaning, ${ }^{4-6}$ anticorrosion, ${ }^{7-9}$ water collection, ${ }^{10-12}$ energy conversation $^{13,14}$ etc. There are many works focused on superhydrophobic epoxy resin (EP) coatings because of the excellent mechanical properties, low manufacturing cost, and their superior adhesion. For example, Zhang et al. ${ }^{15}$ fabricated superhydrophobic EP/PDMS-SiO 2 coatings that were used for self-cleaning and resistance to gamma radiation. Despite the excellent self-cleaning ability and high gamma radiation stability of these materials, a large amount of silica may weaken the mechanical properties of the coating. To further improve the mechanical abrasion, in our previous work, we fabricated superhydrophobic coatings by anchoring functional $\mathrm{SiO}_{2}$ nanoparticles to epoxy resin coatings based on co-curing and thus avoiding phase separation between inorganic nanoparticles and organic epoxy resin. ${ }^{16}$ Though the superhydrophobic epoxy resin nanocomposite coating is strong enough for abrasion resistance, there still exists a problem with the superhydrophobic epoxy resin coatings. The key problem is that epoxy resin is flammable and could generate high heat releasing and lots of smoke particles during combustion, which limits to some extent the wide application of epoxy resins. It is highly desirable to enable epoxy resins to resist both fire and water.

The construction of nanoscale or microscale structures with low surface energy is an effective approach to obtain an artificial superhydrophobic surface. ${ }^{17}$ Using flame retardant particles to create nanoscale or microscale structures will be a benefit for the fabrication of the superhydrophobic surface with flame retardant properties. As an engineering material, the epoxy resin has been widely used in our daily life and the flame retardant properties have received more attention by many researchers. $^{18-25}$ Flame retardants mainly contain halogen- containing and halogen-free flame retardants. Although halogen-containing flame retardants have an obvious flameretardant effect, they have been utilized less than before because of its toxicity and the production of plenty of smoke particles during the combustion. As a halogen-free flame retardant, inorganic nanoflame retardants have gained much more attention and metallic ferrites such as $\mathrm{LaFeO}_{3}{ }^{26}$ $\mathrm{BiFeO}_{3}{ }^{27}$ and $\mathrm{ZnFe}_{2} \mathrm{O}_{4}{ }^{28}$ exhibit outstanding properties as well as fostering a smoke-suppressant effect. Metallic ferrites will decompose into two kinds of metal oxides during combustion and promote a large amount of carbonaceous material covered on the surface of polymers to block the heat and oxygen effectively into the underlying polymers. As a type of metallic ferrite, $\mathrm{CoFe}_{2} \mathrm{O}_{4}$ is considered to be a potential flame retardant material reducing the heat release and it is reported that cobalt species could promote $\mathrm{CO}$ oxidation, decreasing the toxic hazards significantly. ${ }^{29}$ In this work, we prepared hydrophobic $\mathrm{CoFe}_{2} \mathrm{O}_{4}$ particles and added to inherently hydrophilic epoxy resin to fabricate free-standing $\mathrm{CoFe}_{2} \mathrm{O}_{4} / \mathrm{EP}$ monoliths. The addition of hydrophobic and flame-retardant $\mathrm{CoFe}_{2} \mathrm{O}_{4}$ particles will construct a rough surface, which is beneficial for the superhydrophobicity of the monolith surface and also to enables remarkable fire resistance of the EP monoliths.

\section{EXPERIMENTAL SECTION}

Materials and Chemicals. Analytical reagents iron(III) chloride hexahydrate $\left(\mathrm{FeCl}_{3} \cdot 6 \mathrm{H}_{2} \mathrm{O}\right)$, cobalt chloride hexahydrate, polyethylene glycol-400 (PEG), sodium acetate ( $\mathrm{NaAc}$ ), sodium hydroxide $(\mathrm{NaOH})$, and tetraethyl orthosilicate were purchased from Tianjin Kermel Chemical Reagent Company (Tianjin, China). Analytical reagents ethylene glycol (EG) and ethanol $\left(\mathrm{C}_{2} \mathrm{H}_{5} \mathrm{OH}\right)$ were provided 
by Tianjin Fuyu Fine Chemical Company Limited (Tianjin, China) and Anhui Ante Food Company Limited (Suzhou, China), respectively. Ammonia solution and $\mathrm{HCl}$ were obtained from Luoyang Haohua Chemical Reagent Co., Ltd. (Luoyang, China). The PDMS precursor (Sylgard 184) was purchased from Dow Corning. KH560 was purchased from Aladdin. Epoxy resin (EP-446101) was commercially obtained from Zhenjiang Danbao Resin Company Limited (Zhenjiang, China). All the reagents were used as received without further purification.

Preparation of $\mathrm{CoFe}_{2} \mathrm{O}_{4}$ and Superhydrophobic $\mathrm{CoFe}_{2} \mathrm{O}_{4} /$ EP. The $\mathrm{CoFe}_{2} \mathrm{O}_{4}$ nanoparticles were prepared via a typical solvothermal method. Briefly, $2 \mathrm{mmol}$ of $\mathrm{FeCl}_{3} \cdot 6 \mathrm{H}_{2} \mathrm{O}$, and $1 \mathrm{mmol}$ of $\mathrm{CoCl}_{2} \cdot 6 \mathrm{H}_{2} \mathrm{O}$ were dispersed in $50 \mathrm{~mL}$ of $\mathrm{EG}$ and ultrasound applied continuously for $2 \mathrm{~h}$. A certain amount of NaAc was added into the above solution to adjust the $\mathrm{pH}$ value to around 7 , and then stirred for another $30 \mathrm{~min}$ to make sure the solution mixed uniformly. Next, the mixed-solution was transferred to a Teflon autoclave and heated at $200{ }^{\circ} \mathrm{C}$ for $10 \mathrm{~h}$. After the reaction, the mixture was allowed to cool down and the precipitate was filtered. The precipitate was washed several times with distilled water and ethanol, then dried at 60 ${ }^{\circ} \mathrm{C}$ and the $\mathrm{CoFe}_{2} \mathrm{O}_{4}$ nanoparticles were obtained.

$4 \mathrm{~g}$ of $\mathrm{CoFe}_{2} \mathrm{O}_{4}$ was dispersed into $80 \mathrm{~mL}$ deionized water and 320 $\mathrm{mL}$ absolute ethanol and stirred for $20 \mathrm{~min}$. Then $12 \mathrm{~mL}$ of ammonia was added into the above solution and stirred for another $10 \mathrm{~min}$. After that, $12 \mathrm{~mL}$ of TEOS was added as slowly as possible and then refluxed for $12 \mathrm{~h}$. The $\mathrm{SiO}_{2}$-coated $\mathrm{CoFe}_{2} \mathrm{O}_{4}$ particles were obtained by magnetic separation.

In order to turn hydrophilic $\mathrm{CoFe}_{2} \mathrm{O}_{4} @ \mathrm{SiO}_{2}$ particles to hydrophobic ones, perfluorodecyl- $1 \mathrm{H}, 1 \mathrm{H}, 2 \mathrm{H}, 2 \mathrm{H}$-trichlorosilane (FDTS) was used to as a modifier. In a typical synthesis, $0.19 \mathrm{~g}$ of FDTS and $3.8 \mathrm{~g}$ of $\mathrm{CoFe}_{2} \mathrm{O}_{4} @ \mathrm{SiO}_{2}$ were dissolved in $100 \mathrm{~mL}$ of $n$-hexane and refluxed for $3 \mathrm{~h}$ under ultrasound. Finally, the mixture was separated by a magnet, followed by ethanol washing for 3 times and dried at $60{ }^{\circ} \mathrm{C}$.

To get superhydrophobic $\mathrm{CoFe}_{2} \mathrm{O}_{4} / \mathrm{EP}$ items, $40 \mathrm{~g}$ of EP filled with $1.2 \mathrm{~g}$ PDMS, $1.2 \mathrm{~g} \mathrm{KH} 560$, and different amounts of hydrophobic $\mathrm{CoFe}_{2} \mathrm{O}_{4} @ \mathrm{SiO}_{2}$ particles ( 3 and $10 \mathrm{wt} \%$ ) were stirred by a ZYMC200V deaeration mixing mill (Shenzhen Zhongyi Technological Limited company) for $10 \mathrm{~min}$. Then, $10 \mathrm{~g}$ of curing agent was poured into the above mixture and fully mixed for another $90 \mathrm{~s}$. The resultant mixture was cured at $80{ }^{\circ} \mathrm{C}$ for $2 \mathrm{~h}$ to get $\mathrm{CoFe}_{2} \mathrm{O}_{4} @ \mathrm{EP}$ nanocomposite items. Herein, the addition of PDMS and KH560 will lower the item surface energy and improve the compatibility between nanoparticles and EP polymers.

Characterizations. X-ray powder diffraction (XRD) patterns were obtained with an X'Pert Pro diffractometer ( $\mathrm{Cu} \mathrm{K} \alpha$ radiation, $\lambda=$ $0.15418 \mathrm{~nm}$; voltage: $40 \mathrm{kV}$, current: $40 \mathrm{~mA}$ ). A JEM-2100 transmission electron microscope (Japan Electronics Corporation, Japan) was used to analyze the microstructure of nanoparticles. The dispersion state of the nanofillers in the EP matrix was observed with a JSM-7001F scanning electron microscope (Japan Electronics, Japan). A Renishaw inVia confocal microscope (excitation source: $532 \mathrm{~nm}$ laser) was used to record the Raman scattering spectra of the residue chars of EP-matrix nanocomposites. Thermogravimetric analysis (TGA) was conducted with a TGA/DSC3+ thermal analyzer (Mettler Toledo, Switzerland), where about $5.0 \mathrm{mg}$ of the to-be-tested sample was tested in a nitrogen atmosphere from room temperature to $800{ }^{\circ} \mathrm{C}$ at a heating rate of $10{ }^{\circ} \mathrm{C} / \mathrm{min}$. The combustion performance of EP and EP-matrix nanocomposites was evaluated by cone calorimetry tests (Fire Testing Technology, UK). According to the procedures described in ISO-5660, the to-be-tested specimens with a dimension of $100 \times 100 \times 3 \mathrm{~mm}^{3}$ were exposed to $35 \mathrm{~kW} \cdot \mathrm{m}^{-2}$ heat flux. The water and other liquid contact angle (CA) tests were measured on a DSA-100S optical contact-angle system (Kruss Company, Germany) at room temperature. The volume of all the liquids was 4 or $10 \mu \mathrm{L}$ when the CA was measured. All the optical photos were taken with a digital camera (NIKON, P600).

Durability Test. UV-accelerated weathering tester (UV-II, Shanghai Pushen Chemical Machinery; Shanghai, China) was used to evaluate the UV resistance of the as-prepared items. $\mathrm{HCl}(\mathrm{pH}=1)$ and $\mathrm{NaOH}(\mathrm{pH}=14)$ solution was employed to evaluate the durability of the prepared $\mathrm{CoFe}_{2} \mathrm{O}_{4} / \mathrm{EP}$ in highly acidic and alkaline environments. The corrosive droplet $(10 \mu \mathrm{L})$ on the surface of the prepared sample was used to measure the CA.

The sandpaper abrasion tests were also carried out to test the durability of superhydrophobicity. The obtained monolith was placed face-down on the sandpaper (standard glass paper, grit no. 100) under a weight of $200 \mathrm{~g}$ and moved for $10 \mathrm{~cm}$, and then the sample was rotated at $90^{\circ}$ with respect to the sandpaper and then moved $10 \mathrm{~cm}$. This process is defined as an abrasion cycle, which guarantees the surface is abraded longitudinally.

\section{RESULTS AND DISCUSSION}

Figure 1 illustrates the preparation mechanism of superhydrophobic $\mathrm{CoFe}_{2} \mathrm{O}_{4} / \mathrm{EP}$. $\mathrm{CoFe}_{2} \mathrm{O}_{4}$ nanoparticles were

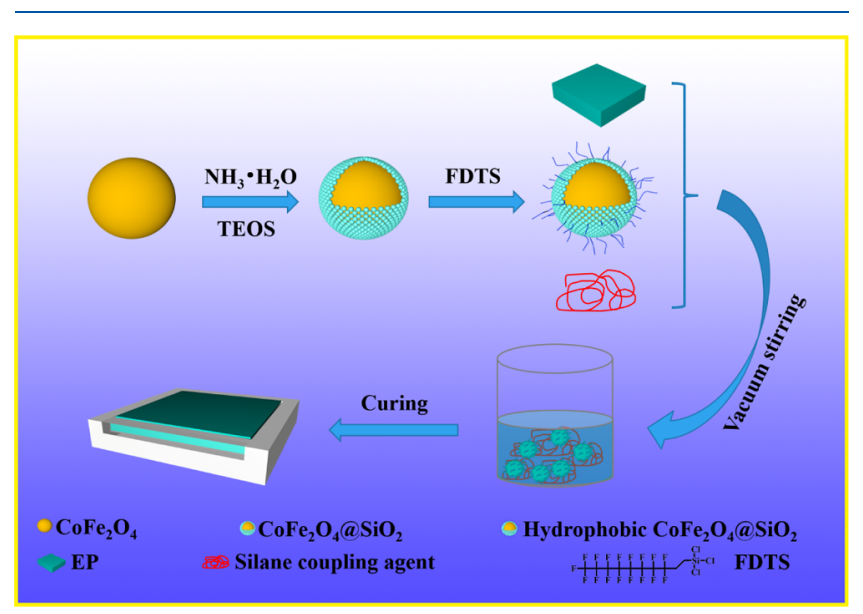

Figure 1. Schematic illustration of the fabrication process of superhydrophobic $\mathrm{CoFe}_{2} \mathrm{O}_{4} / \mathrm{EP}$.

coated by a layer of silica and then modified by a silane coupling agent. After modification, hydrophilic $\mathrm{CoFe}_{2} \mathrm{O}_{4}$ nanoparticles turned to hydrophobic. Subsequently, surface modified $\mathrm{CoFe}_{2} \mathrm{O}_{4}$ was added to EP with the curing agent and cured to get the superhydrophobic $\mathrm{CoFe}_{2} \mathrm{O}_{4} / \mathrm{EP}$ monoliths.

Figure 2a shows the XRD pattern and it can be seen that the diffraction peaks of $\mathrm{CoFe}_{2} \mathrm{O}_{4}$ nanoparticles match well with the standard spinel $\mathrm{CoFe}_{2} \mathrm{O}_{4}$, according to JCPDS no. 22-1086, and there exists several main peaks centered at $2 \theta$ of around $30.2^{\circ}, 35.6^{\circ}, 37.2^{\circ}, 43.2^{\circ}, 53.8^{\circ}, 57.2^{\circ}$, and $62.7^{\circ}$, belonging to the planes of (220), (311), (222), (400), (422), (511), and (440), respectively. ${ }^{30}$ After coating by a layer of $\mathrm{SiO}_{2}$, the obtained $\mathrm{CoFe}_{2} \mathrm{O}_{4} @ \mathrm{SiO}_{2}$ particles present similar XRD patterns to $\mathrm{CoFe}_{2} \mathrm{O}_{4}$ except for a new broad peak at $2 \theta$ of $20-25^{\circ}$ attributed to amorphous silica. ${ }^{31}$ From the transmission electron microscopy (TEM) image (Figure $2 \mathrm{~b}$ ), it can be seen that $\mathrm{CoFe}_{2} \mathrm{O}_{4}$ nanoparticles were coated with a layer of $\mathrm{SiO}_{2}$ and the $\mathrm{SiO}_{2}$ thickness is about $50 \mathrm{~nm}$. Figure $2 \mathrm{c}$ shows the dispersion of the as-prepared $\mathrm{CoFe}_{2} \mathrm{O}_{4} @ \mathrm{SiO}_{2}$ nanoparticles in water. It is clear that $\mathrm{CoFe}_{2} \mathrm{O}_{4} @ \mathrm{SiO}_{2}$ can disperse in water well before modification by FDTS. However, after surface modification with FDTS, the $\mathrm{CoFe}_{2} \mathrm{O}_{4} @ \mathrm{SiO}_{2}$ nanoparticles turn to hydrophobic and cannot be dispersed in water. In addition, the chemical composition was further verified by energy dispersive X-ray spectroscopy (EDS) (Figure $2 \mathrm{~d}$ ) and mapping (Figure $2 \mathrm{e}-\mathrm{j}$ ). Fluorine appears in the composition, indicating that the $\mathrm{CoFe}_{2} \mathrm{O}_{4} @ \mathrm{SiO}_{2}$ nanoparticles were successfully modified by FDTS. The surface morphology of the superhydrophobic $\mathrm{CoFe}_{2} \mathrm{O}_{4} / \mathrm{EP}$ surface is 


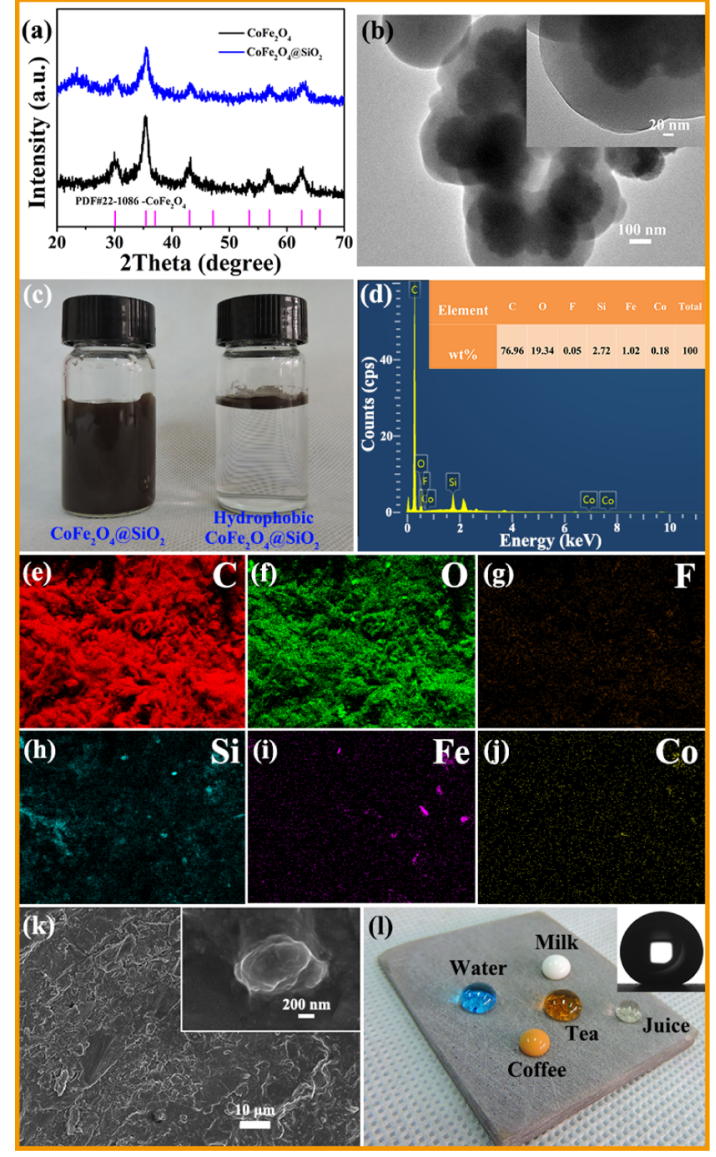

Figure 2. (a) XRD patterns of $\mathrm{CoFe}_{2} \mathrm{O}_{4}, \mathrm{CoFe}_{2} \mathrm{O}_{4} @ \mathrm{SiO}_{2}$ nanoparticles. (b) TEM image of $\mathrm{CoFe}_{2} \mathrm{O}_{4} @ \mathrm{SiO}_{2}$ nanoparticles. (c) Photo of $\mathrm{CoFe}_{2} \mathrm{O}_{4} @ \mathrm{SiO}_{2}$ and hydrophobic $\mathrm{CoFe}_{2} \mathrm{O}_{4} @ \mathrm{SiO}_{2}$ particles dispersed in water. (d) EDS spectra of the hydrophobic $\mathrm{CoFe}_{2} \mathrm{O}_{4} /$ EP. (e-j) Mapping of the hydrophobic $\mathrm{CoFe}_{2} \mathrm{O}_{4} /$ EP. (k) SEM images of different magnifications of superhydrophobic $\mathrm{CoFe}_{2} \mathrm{O}_{4} / \mathrm{EP}$. (1) Optical images of liquid-resistant tests on the surface of $\mathrm{CoFe}_{2} \mathrm{O}_{4} /$ EP.

shown in Figure 2k. From the SEM image it can be seen that the surface is rough with a hierarchical micro/nanostructure that is necessary for superhydrophobicity. In addition, the cross-sections of the $\mathrm{EP}$ and $\mathrm{CoFe}_{2} \mathrm{O}_{4} / \mathrm{EP}$ nanocomposite surface were observed by SEM observation in Figure $\mathrm{S} 1$ in the Supporting Information. It can be seen that the $\mathrm{CoFe}_{2} \mathrm{O}_{4} @$ $\mathrm{SiO}_{2}$ particles have a good dispersion in the EP matrix. Water droplets sitting on the obtained surface form a near-spherical shape with a CA of about $157^{\circ}$. The surface also shows to be highly repellent to many liquids such as tea, coffee, milk, juice droplet, etc (Figure 21).

Thermal stability of pure EP was conducted by TG analysis as shown in Figure 3a, and the relative parameters such as $T_{\text {onest }}$ and $T_{\max }$ are summarized in Tables S1 and S2. It could be found that $\mathrm{CoFe}_{2} \mathrm{O}_{4} @ \mathrm{SiO}_{2}$ composites show extremely similar decomposition behavior to that of pristine EP, which has mainly one-stage decomposition. The $T_{\text {onest }}$ of $\mathrm{CoFe}_{2} \mathrm{O}_{4} /$ EP with different $\mathrm{CoFe}_{2} \mathrm{O}_{4}$ nanoparticles adding shows a reduced value compared to the pure sample, mainly because of the higher thermal stability of $\mathrm{CoFe}_{2} \mathrm{O}_{4}$. As for the residues at $800{ }^{\circ} \mathrm{C}$, pristine EP has little char residues. The $\mathrm{CoFe}_{2} \mathrm{O}_{4} / \mathrm{EP}$ composite exhibits much higher residual chars at the evaluated temperature, and the value is 16.3 and $31.5 \mathrm{wt} \%$, respectively.
Hence, it is believed that the introduction of $\mathrm{CoFe}_{2} \mathrm{O}_{4}$ into the EP matrix promotes the formation of proactive char layers on the surface of the polymer, which modifies the path of heat and oxygen ingress to enhance the fire resistance.

Cone calorimetry tests are commonly employed to evaluate the fire resistance of polymers in real-world fire situations. As shown in Figure $3 b$, the HRR curves of pure EP showed narrower peaks with a higher PHRR value of about 1128.7 $\mathrm{kW} / \mathrm{m}^{2}$, demonstrating violent burning. Different from the pristine EP, the HRR curves of $\mathrm{CoFe}_{2} \mathrm{O}_{4} / \mathrm{EP}$ with different $\mathrm{CoFe}_{2} \mathrm{O}_{4}$ nanoparticles presented broader peaks, and the PHRR value decreased significantly, indicating better flame retardancy. $\mathrm{CoFe}_{2} \mathrm{O}_{4} / \mathrm{EP}$ with $10 \mathrm{wt} \% \mathrm{CoFe}_{2} \mathrm{O}_{4}$ addition has the widest peak with the lowest PHRR value of $681.9 \mathrm{~kW} / \mathrm{m}^{2}$, indicating excellent fire resistance. It is well known that smoke is a potential killer from a fire; it is necessary and significant to suppress smoke formation during polymer combustion. As shown in Figure 3c, the PSPR value of $\mathrm{CoFe}_{2} \mathrm{O}_{4} / \mathrm{EP}$ decreases with the addition of $\mathrm{CoFe}_{2} \mathrm{O}_{4}$, especially at $10 \mathrm{wt} \%$. From Figure $3 \mathrm{~d}$, it is worth noting that $\mathrm{CoFe}_{2} \mathrm{O}_{4}$ is a potential material to reduce the toxicity especially toxic $\mathrm{CO}$. The COP value of the sample with $10 \mathrm{wt} \% \mathrm{CoFe}_{2} \mathrm{O}_{4}$ addition decreases significantly by $61.3 \%$, compared to pure $\mathrm{EP}$, indicating that $\mathrm{CoFe}_{2} \mathrm{O}_{4}$ endows EP with excellent $\mathrm{CO}$ abatement. As by the weight curves (Figure $3 \mathrm{e}$ ) from the polymers, it is found that the addition of $\mathrm{CoFe}_{2} \mathrm{O}_{4}$ could improve the residue weight significantly after burning tests. It is expected that $\mathrm{CoFe}_{2} \mathrm{O}_{4}$ plays an important role in catalyzing EP decomposition to form a protective char layer and a cover on the surface of EP.

The digital photos and SEM images of the char residues of $\mathrm{EP}$ and $\mathrm{CoFe}_{2} \mathrm{O}_{4} / \mathrm{EP}$ were studied to analyze the potential mechanism of flame retardancy. As shown in Figure 4a, it is found that pristine EP has less char residues, consistent with the poor flame-retardant effect. Different from EP, the char layers of $\mathrm{CoFe}_{2} \mathrm{O}_{4} / \mathrm{EP}$ are more continuous and denser, which helps prevent oxygen and heat to permeate into the underlying layer. SEM images (Figure $4 \mathrm{~b}$ ) show that the pure EP after burning has a loose char structure with more cracks and holes, which is not helpful to form the good barrier layer to block the heat, oxygen, and flammable gases. Whilst, $\mathrm{CoFe}_{2} \mathrm{O}_{4} / \mathrm{EP}$ has more continuous and firmer protective char layers, contributing to modify the path of heat and oxygen, which correspond with excellent flame retardantperformance. From the high magnification SEM image, it is clear that many nanocrystals and nanospheres exist in the condensed phase, which could strengthen the char layers. It is believed that PDMS is decomposed into nanosilica while hydrophobic $\mathrm{CoFe}_{2} \mathrm{O}_{4} @$ $\mathrm{SiO}_{2}$ nanofillers grow during the combustion process; the regrowth $\mathrm{CoFe}_{2} \mathrm{O}_{4}$ and nanosilica could promote the formation of the proactive char layers on the surface of the polymer, which exchange the path of heat and oxygen to enhance thermal stability. ${ }^{32}$

The components of the char of EP after combustion were detected by XRD analysis, with the aim of further understanding the potential mechanism of the flame retardant. As shown in Figure 5a, pristine EP presents a broad peak at $2 \theta$ of $23^{\circ}$, which belongs to the (002) plane of graphite. ${ }^{33}$ The composition of the $\mathrm{CoFe}_{2} \mathrm{O}_{4} / \mathrm{EP}$ residue after burning consists of $\mathrm{CoFe}_{2} \mathrm{O}_{4}$ nanoparticles, which covers the EP surface to strengthen the char layers. In order to evaluate the char structure of EP and $\mathrm{CoFe}_{2} \mathrm{O}_{4} / \mathrm{EP}$, Raman spectra were obtained. As shown in Figure $5 \mathrm{~b}-\mathrm{d}$, all the polymers have two bands, which belong to the D $\left(1350-1380 \mathrm{~cm}^{-1}\right)$ and G 

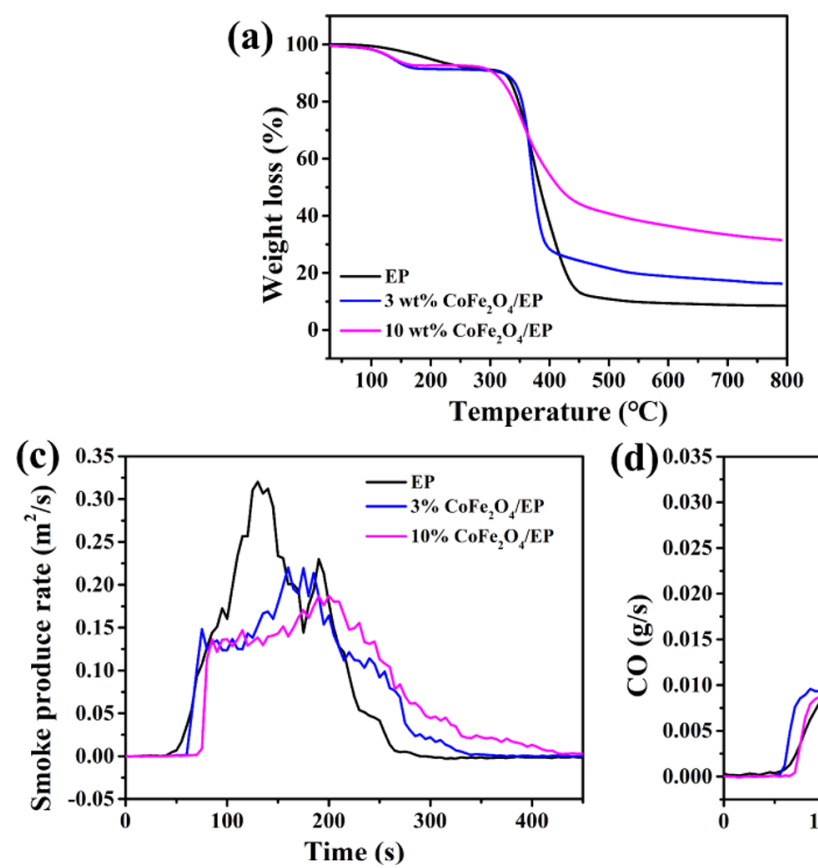
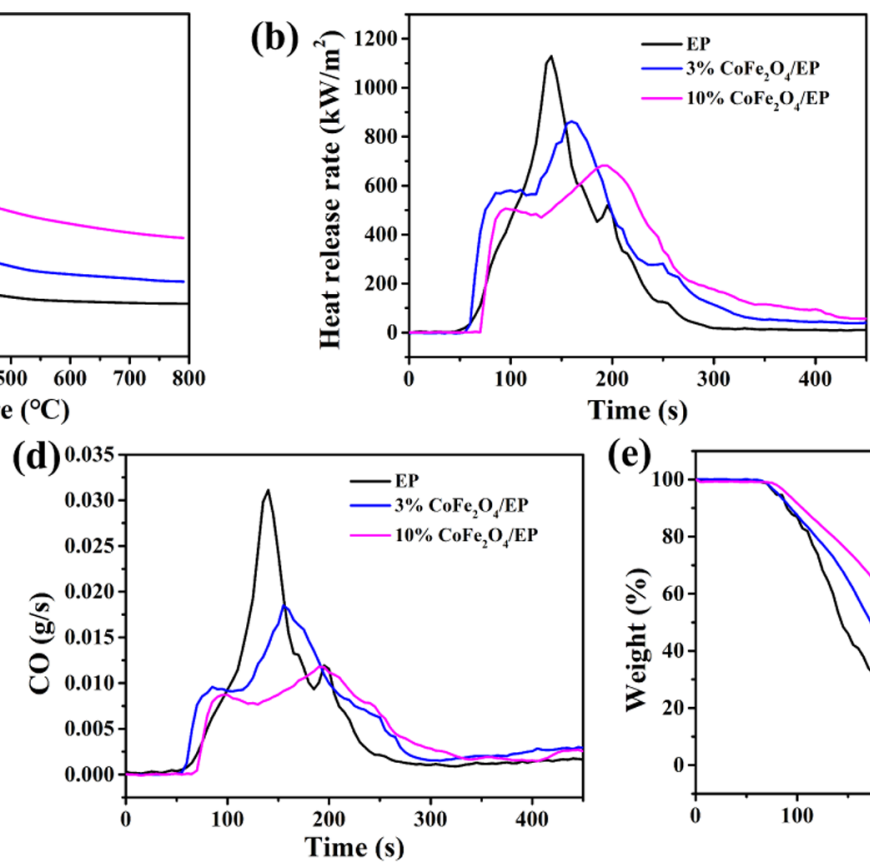

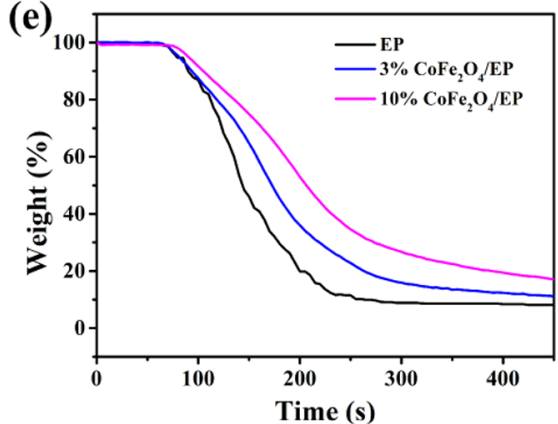

Figure 3. (a) TG curves of EP and its nanocomposites under a $\mathrm{N}_{2}$ atmosphere. (b) HRR, (c) SPR, and (d) COP, (e) weight curves of EP and $\mathrm{CoFe}_{2} \mathrm{O}_{4} / \mathrm{EP}$ nanocomposites obtained from the cone calorimetry tests.

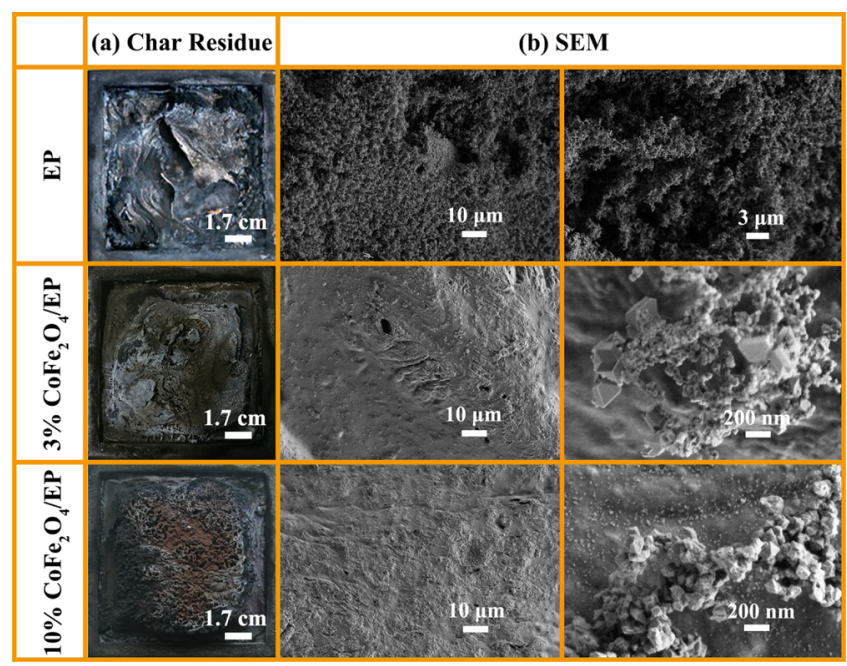

Figure 4. $\mathrm{EP}$ and $\mathrm{CoFe}_{2} \mathrm{O}_{4} / \mathrm{EP}$ residual char surface analysis. (a) Digital photos; (b) SEM images.

(1580-1600 $\mathrm{cm}^{-1}$ ) band of graphite. Normally, the $I_{\mathrm{D}} / I_{\mathrm{G}}$ value is used to evaluate the graphitization degree of the char residues. It could be observed that pure EP had the highest value of $I_{\mathrm{D}} / I_{\mathrm{G}}$, corresponding to the less stable char layer. Different from pure EPs, $\mathrm{CoFe}_{2} \mathrm{O}_{4} /$ EP-tested char have lower $I_{\mathrm{D}} / I_{\mathrm{G}}$ values, corresponding to the higher graphitization degree of the char layer. Especially, when the addition of $\mathrm{CoFe}_{2} \mathrm{O}_{4}$ reaches $10 \%$, the nanocomposite exhibits lower $I_{\mathrm{D}} / I_{\mathrm{G}}$ values, indicating high stability of the residual char, which is consistent with excellent flame retardancy.

Mechanical friction leads to the damage of the fragile micro/ nano surface structures that are necessary for the superhydrophobic property, hence, durability is an important factor for practical applications of superhydrophobic materials. ${ }^{34-36}$ In this work, the mechanical durability and chemical stability were investigated and it was found that the monolith surface
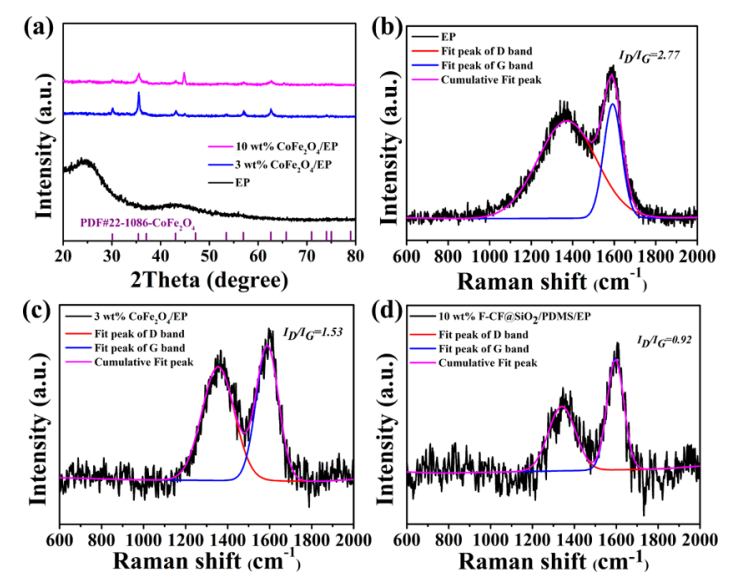

Figure 5. XRD patterns (a) and Raman curves $(b-d)$ of the char residues of $\mathrm{EP}$ and $\mathrm{CoFe}_{2} \mathrm{O}_{4} / \mathrm{EP}$.

could retain superhydrophobicity after a $2000 \mathrm{~cm}$ sandpaper abrasion, knife scratch test, high pressure, acidic/alkali corrosion and long-term UV irradiation. As shown in Figure $6 \mathrm{a}, \mathrm{b}$, after 300 cycles of abrasion, the $\mathrm{CoFe}_{2} \mathrm{O}_{4} / \mathrm{EP}$ bulk surfaces still retained superhydrophobicity and the weight loss was less than $1 \%$, indicating the extremely excellent robustness. In addition, the bulk surface is strong enough to stand knife scratches and sustain its superhydrophobicity since the monolith surface and interior has the same chemical composition and microstructure. When the surface uppermost layer is destroyed upon scrape abrasion, the newly exposed surface is also water-repellent, thereby making the superhydrophobicity permanent. As shown in Figure 6d,e and Video S1 in the Supporting Information, water droplets immediately roll off without wetting the destroyed surface. Figure $6 \mathrm{f}, \mathrm{g}$ and Video S2 in the Supporting Information show that the asprepared $\mathrm{CoFe}_{2} \mathrm{O}_{4} / \mathrm{EP}$ monolith is exposed to the smooth steel plate under a pressure of $200 \mathrm{~N}$. After the pressure is 


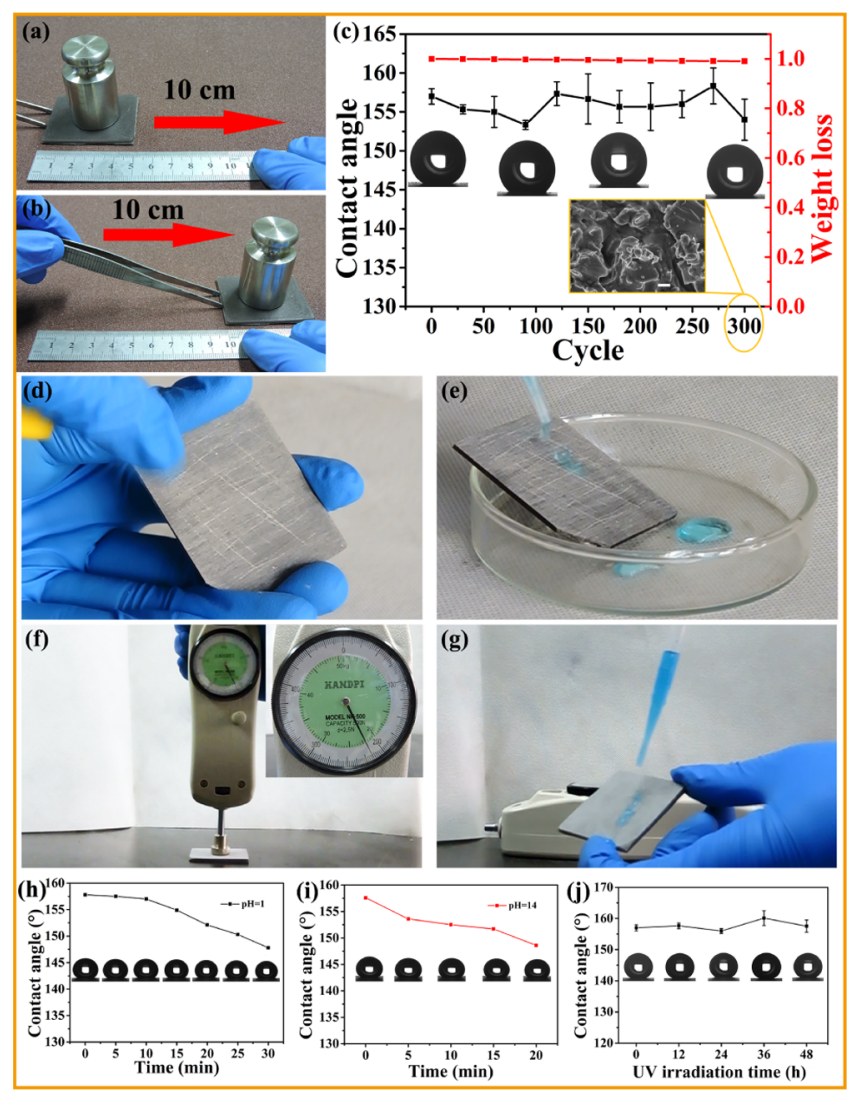

Figure 6. Durability of the superhydrophobic $\mathrm{CoFe}_{2} \mathrm{O}_{4} / \mathrm{EP}$ monolith. $(\mathrm{a}, \mathrm{b})$ Sandpaper abrasion test; (c) plot of mechanical abrasion cycles with water CAs and weight loss after each 30 abrasion tests (Inset: SEM image abrasion test after 300 cycles of abrasion and photos of WCA). (d) Scratched by a knife; (e) the monolith retains superhydrophobicity after damage. (f,g) Newton meter press test at $200 \mathrm{~N}$ and water bouncing on the surface after the pressure is released. CAs as a function of contact time with acidic (h) and alkali (i) liquids on the superhydrophobic $\mathrm{CoFe}_{2} \mathrm{O}_{4} / \mathrm{EP}$ surfaces. ( $\mathrm{j}$ ) Relationship between water CA and UV irradiation time.

released, the monolith still keeps its superhydrophobic properties, demonstrating good mechanical robustness.

Figure $6 \mathrm{~h}$,i show the CA as a function of continuous contact time with extremely corrosive liquids. The monolith surfaces still keep their superhydrophobic properties after $25 \mathrm{~min}$ acid and $15 \mathrm{~min}$ alkali bath, respectively. Superhydrophobic surfaces are usually subject to UV-light exposure because the presence of large quantities of organic components leads to the oxidation and formation of hydrophilic groups. Herein, even after $48 \mathrm{~h}$ of continuous UV irradiation, the CA is still higher than $150^{\circ}$ without significant change, as shown in Figure 6j, which demonstrates that the prepared monolith has good UV resistance.

In this work, the obtained monolith surface also shows superhydrophobicity in air or under oil. As shown in Figure $7 a-d$ and Video S3 in the Supporting Information, water drops form nearly spherical drops and completely leave the surface without wetting the surface no matter whether in air or under oil. It is well known that superhydrophobic surfaces have good self-cleaning properties in air. When a water droplet falls on a superhydrophobic surface, it promptly rolls off the surface collecting dirt along the way. Herein, the robust monolith surface demonstrates excellent self-cleaning property even under oil. Figure $7 \mathrm{e}-1$ and Video $\mathrm{S} 4$ in the Supporting Information shows that water droplets can carry the dust off the surface immediately and leave the surface clean in air and the dirt removal property in oil is similar to that in air. The dust on the monolith surface immersed in oil can also be easily taken away, which is very important for the robust superhydrophobic surface used as self-cleaning materials in practical applications.

\section{CONCLUSIONS}

In summary, we have successfully fabricated flame retardant and superhydrophobic $\mathrm{CoFe}_{2} \mathrm{O}_{4} / \mathrm{EP}$ monoliths by integrating flame-retardant $\mathrm{CoFe}_{2} \mathrm{O}_{4}$ into $\mathrm{EP}$ polymers. The monoliths present excellent mechanical properties and good abrasion resistance. After 300 cycle abrasion, the $\mathrm{CoFe}_{2} \mathrm{O}_{4} / \mathrm{EP}$ monolith surfaces still retained superhydrophobicity and the weight loss was less than $1 \%$, indicating excellent robustness. The monolith shows robust superhydrophobic properties to harsh environments such as strong corrosion, exposure under UV irradiation and external mechanical pressure. It is expected that the good flame retardant and smoke suppressing properties will make the superhydrophobic epoxy resin have wider applications.

\section{ASSOCIATED CONTENT}

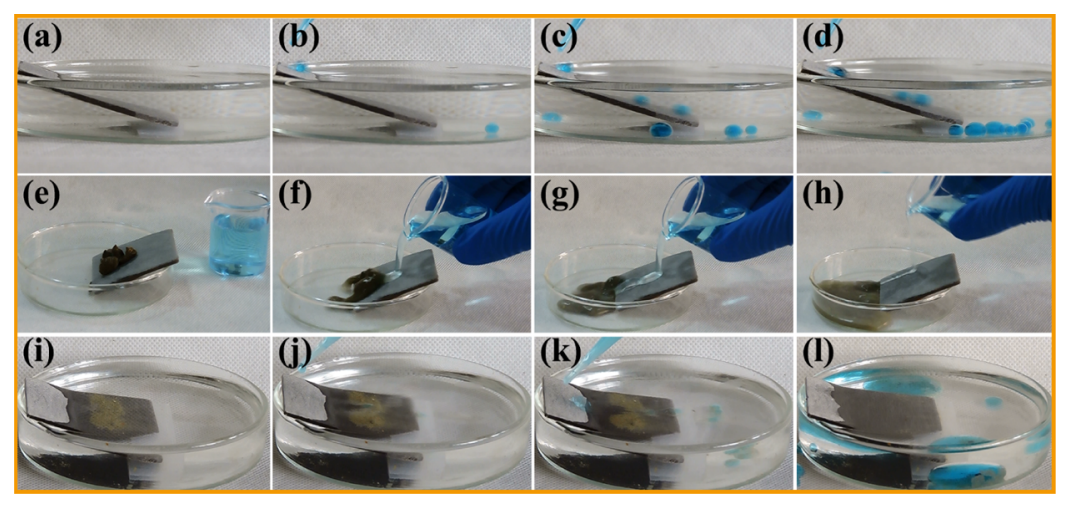

Figure 7. $(\mathrm{a}-\mathrm{d})$ superhydrophobicity of the $\mathrm{CoFe}_{2} \mathrm{O}_{4} / \mathrm{EP}$ surfaces both in air and under oil. Self-cleaning properties of the $\mathrm{CoFe}_{2} \mathrm{O}_{4} / \mathrm{EP}$ surfaces in air $(\mathrm{e}-\mathrm{h})$ and under oil $(\mathrm{i}-\mathrm{l})$. 
SEM imaging of the cross-sections of the EP and $\mathrm{CoFe}_{2} \mathrm{O}_{4} / \mathrm{EP}$ nanocomposite; thermal analysis data of the $\mathrm{EP}$ and $\mathrm{CoFe}_{2} \mathrm{O}_{4} / \mathrm{EP}$ nanocomposite under nitrogen atmosphere; and burning parameters of the $\mathrm{EP}$ and $\mathrm{CoFe}_{2} \mathrm{O}_{4} / \mathrm{EP}$ nanocomposite (PDF)

Knife-scratch testing on the surface of superhydrophobic $\mathrm{CoFe}_{2} \mathrm{O}_{4} / \mathrm{EP}$ (MP4)

Pressure testing on the surface of superhydrophobic $\mathrm{CoFe}_{2} \mathrm{O}_{4} / \mathrm{EP}$ (MP4)

The monolith surface shows superhydrophobicity in air or under oil (MP4)

Self-cleaning properties of the monolith surface in air or under oil (MP4)

\section{AUTHOR INFORMATION}

\section{Corresponding Author}

*E-mail: zx@henu.edu.cn.

\section{ORCID $\odot$}

Xia Zhang: 0000-0003-2496-8780

Zhiwei Li: 0000-0003-2161-8486

\section{Author Contributions}

${ }^{\S}$ X.W. and Q.Z. contributed equally.

\section{Notes}

The authors declare no competing financial interest.

\section{ACKNOWLEDGMENTS}

This project is supported by the National Natural Science Foundation of China (grant no. 51875173) and the innovative talents promotion plan of Henan province (20HASTIT003). I.P.P. acknowledges Engineering and Physical Sciences Research Council, UK (EP/L015862/1) for the financial support.

\section{REFERENCES}

(1) Gore, P. M.; Kandasubramanian, B. Heterogeneous wettable cotton based superhydrophobic Janus biofabric engineered with PLA/ functionalized-organoclay microfibers for efficient oil-water separation. J. Mater. Chem. A 2018, 6, 7457-7479.

(2) Cao, G.; Zhang, W.; Jia, Z.; Liu, F.; Yang, H.; Yu, Q.; Wang, Y.; Di, X.; Wang, C.; Ho, S.-H. Dually prewetted underwater superoleophobic and under oil superhydrophobic fabric for successive separation of light oil/water/heavy oil three-phase mixtures. ACS Appl. Mater. Interfaces 2017, 9, 36368-36376.

(3) Liu, M.; Hou, Y.; Li, J.; Guo, Z. Stable superwetting meshes for on-demand separation of immiscible oil/water mixtures and emulsions. Langmuir 2017, 33, 3702-3710.

(4) Lin, J.; Zheng, C.; Ye, W.; Wang, H.; Feng, D.; Li, Q.; Huan, B. A facile dip-coating approach to prepare $\mathrm{SiO}_{2}$ /fluoropolymer coating for superhydrophobic and superoleophobic fabrics with self-cleaning property. J. Appl. Polym. Sci. 2015, 132, 41458.

(5) Cao, C.; Ge, M.; Huang, J.; Li, S.; Deng, S.; Zhang, S.; Chen, Z.; Zhang, K.; Al-Deyab, S. S.; Lai, Y. Robust fluorine-free superhydrophobic PDMS-ormosil@fabrics for highly effective self-cleaning and efficient oil-water separation. J. Mater. Chem. A 2016, 4, 1217912187.

(6) Chen, B.; Qiu, J.; Sakai, E.; Kanazawa, N.; Liang, R.; Feng, H. Robust and Superhydrophobic Surface Modification by a "Paint + Adhesive" Method: Applications in Self-Cleaning after Oil Contamination and Oil-Water Separation. ACS Appl. Mater. Interfaces 2016, 8, 17659-17667.

(7) Xiao, F.; Yuan, S.; Liang, B.; Li, G.; Pehkonen, S. O.; Zhang, T. Superhydrophobic $\mathrm{CuO}$ nanoneedle-covered copper surfaces for anticorrosion. J. Mater. Chem. A 2015, 3, 4374-4388.
(8) Wang, N.; Xiong, D.; Deng, Y.; Shi, Y.; Wang, K. Mechanically robust superhydrophobic steel surface with anti-icing, UV-durability, and corrosion resistance properties. ACS Appl. Mater. Interfaces 2015, $7,6260-6272$.

(9) Wang, N.; Xiong, D. Superhydrophobic membranes on metal substrate and their corrosion protection in different corrosive media. Appl. Surf. Sci. 2014, 305, 603-608.

(10) Wang, Y.; Wang, X.; Lai, C.; Hu, H.; Kong, Y.; Fei, B.; Xin, J. $\mathrm{H}$. Biomimetic water-collecting fabric with light-induced superhydrophilic bumps. ACS Appl. Mater. Interfaces 2016, 8, 2950-2960.

(11) Jiang, T.; Guo, Z.; Liu, W. Biomimetic superoleophobic surfaces: focusing on their fabrication and applications. J. Mater. Chem. A 2015, 3, 1811-1827.

(12) Zheng, Y.; Bai, H.; Huang, Z.; Tian, X.; Nie, F.-Q.; Zhao, Y.; Zhai, J.; Jiang, L. Directional water collection on wetted spider silk. Nature 2010, 463, 640-643.

(13) Hao, C.; Liu, Y.; Chen, X.; Li, J.; Zhang, M.; Zhao, Y.; Wang, Z. Bioinspired interfacial materials with enhanced drop mobility: from fundamentals to multifunctional applications. Small 2016, 12, 18251839.

(14) Luo, Y.; Li, J.; Zhu, J.; Zhao, Y.; Gao, X. Fabrication of condensate microdrop self-propelling porous films of cerium oxide nanoparticles on copper surfaces. Angew. Chem., Int. Ed. 2015, 54, 4876-4879.

(15) Zhang, Y.; Ren, F.; Liu, Y. A superhydrophobic EP/PDMS nanocomposite coating with high gamma radiation stability. Appl. Surf. Sci. 2018, 436, 405-410.

(16) Zhi, D.; Wang, H.; Jiang, D.; Parkin, I. P.; Zhang, X. Reactive silica nanoparticles turn epoxy coating from hydrophilic to superrobust superhydrophobic. RSC Adv. 2019, 9, 12547-12554.

(17) Rostami, A.; Sharifnia, S. Fabrication of robust and durable superhydrophobic fiberglass fabrics for oil-water separation based on self-assembly of novel N-TESPO and N-TESPS reagents. J. Mater. Chem. A 2017, 5, 680-688.

(18) Fang, F.; Ran, S.; Fang, Z.; Song, P.; Wang, H. Improved flame resistance and thermo-mechanical properties of epoxy resin nanocomposites from functionalized graphene oxide via self-assembly in water. Composites, Part B 2019, 165, 406-416.

(19) Guo, W.; Yu, B.; Yuan, Y.; Song, L.; Hu, Y. In situ preparation of reduced graphene oxide/DOPO-based phosphonamidate hybrids towards high-performance epoxy nanocomposites. Composites, Part B 2017, 123, 154-164.

(20) Yu, B.; Shi, Y.; Yuan, B.; Qiu, S.; Xing, W.; Hu, W.; Song, L.; Lo, S.; Hu, Y. Enhanced thermal and flame retardant properties of flame-retardant-wrapped graphene/epoxy resin nanocomposites. $J$. Mater. Chem. A 2015, 3, 8034-8044.

(21) Li, Z.; González, A. J.; Heeralal, V. B.; Wang, D.-Y. Covalent assembly of MCM-41 nanospheres on graphene oxide for improving fire retardancy and mechanical property of epoxy resin. Composites, Part B 2018, 138, 101-112.

(22) Kalali, E. N.; Wang, X.; Wang, D.-Y. Functionalized layered double hydroxide-based epoxy nanocomposites with improved flame retardancy and mechanical properties. J. Mater. Chem. A 2015, 3, $6819-6826$

(23) Yu, B.; Xing, W.; Guo, W.; Qiu, S.; Wang, X.; Lo, S.; Hu, Y. Thermal exfoliation of hexagonal boron nitride for effective enhancements on thermal stability, flame retardancy and smoke suppression of epoxy resin nanocomposites via sol-gel process. J. Mater. Chem. A 2016, 4, 7330-7340.

(24) Rajaei, M.; Kim, N. K.; Bickerton, S.; Bhattacharyya, D. A comparative study on effects of natural and synthesised nano-clays on the fire and mechanical properties of epoxy composites. Composites, Part B 2019, 165, 65-74.

(25) Feng, X.; Xing, W.; Song, L.; Hu, Y. In situ synthesis of a $\mathrm{MoS}_{2} / \mathrm{CoOOH}$ hybrid by a facile wet chemical method and the catalytic oxidation of $\mathrm{CO}$ in epoxy resin during decomposition. $J$. Mater. Chem. A 2014, 2, 13299.

(26) Wang, L.; Yang, W.; Wang, B.; Wu, Y.; Hu, Y.; Song, L.; Yuen, R. K. K. The Impact of Metal Oxides on the Combustion Behavior of 
Ethylene-Vinyl Acetate Coploymers Containing an Intumenscent Flame Retardant. Ind. Eng. Chem. Res. 2012, 51, 7884-7890.

(27) An, J.; Zhu, L.; Wang, N.; Song, Z.; Yang, Z.; Du, D.; Tang, H. Photo-Fenton like degradation of tetrabromobisphenol A with graphene $\mathrm{BiFeO}_{3}$ composite as a catalyst. Chem. Eng. J. 2013, 219, 225-237.

(28) Zhang, Q.; Li, Z.; Li, X.; Yu, L.; Zhang, Z.; Wu, Z. Zinc ferrite nanoparticle decorated boron nitride nanosheet: Preparation, magnetic field arrangement, and flame retardancy. Chem. Eng. J. 2019, 356, 680-692.

(29) Zhang, J.; Kong, Q.; Yang, L.; Wang, D.-Y. Few layered $\mathrm{Co}(\mathrm{OH})_{2}$ ultrathin nanosheet-based polyurethane nanocomposites with reduced fire hazard: from eco-friendly flame retardance to sustainable recycling. Green Chem. 2016, 18, 3066-3074.

(30) Chen, L.; Ding, D.; Liu, C.; Cai, H.; Qu, Y.; Yang, S.; Gao, Y.; Cai, T. Degradation of norfloxacin by $\mathrm{CoFe}_{2} \mathrm{O}_{4}-\mathrm{GO}$ composite coupled with peroxymonosulfate: A comparative study and mechanistic consideration. Chem. Eng. J. 2018, 334, 273-284.

(31) Ahmadpoor, F.; Shojaosadati, S. A.; Delavari, H. H.; Christiansen, G.; Saber, R. Synthesis of $\mathrm{Fe}_{5} \mathrm{C}_{2} @ \mathrm{SiO}_{2}$ core@shell nanoparticles as a potential candidate for biomedical application. Mater. Res. Express 2018, 5, 055038.

(32) Zhang, Q.; Li, Z.; Li, X.; Yu, L.; Zhang, Z.; Wu, Z. Preparation of cobalt ferrite nanoparticle-decorated boron nitride nanosheet flame retardant and its flame retardancy in epoxy resin. Nano 2019, 14, 1950063.

(33) Yu, B.; Xing, W.; Guo, W.; Qiu, S.; Wang, X.; Lo, S.; Hu, Y. Thermal exfoliation of hexagonal boron nitride for effective enhancements on thermal stability, flame retardancy and smoke suppression of epoxy resin nanocomposites via sol-gel process. $J$. Mater. Chem. A 2016, 4, 7330-7340.

(34) Hou, Y.; Wang, Z.; Guo, J.; Shen, H.; Zhang, H.; Zhao, N.; Zhao, Y.; Chen, L.; Liang, S.; Jin, Y.; Xu, J. Facile fabrication of robust superhydrophobic porous materials and their application in oil/water separation. J. Mater. Chem. A 2015, 3, 23252-23260.

(35) Lu, Y.; Sathasivam, S.; Song, J.; Crick, C. R.; Carmalt, C. J.; Parkin, I. P. Robust self-cleaning surfaces that function when exposed to either air or oil. Science 2015, 347, 1132-1135.

(36) Zhi, D.; Lu, Y.; Sathasivam, S.; Parkin, I. P.; Zhang, X. Largescale fabrication of translucent and repairable superhydrophobic spray coatings with remarkable mechanical, chemical durability and UV resistance. J. Mater. Chem. A 2017, 5, 10622-10631. 